\title{
Aperture averaging effects on the probability density of irradiance fluctuations in moderate-to-strong turbulence
}

\author{
Frida Strömqvist Vetelino, Cynthia Young, Larry Andrews, and Jaume Recolons
}

\begin{abstract}
The lognormal (LN) and gamma-gamma (GG) distributions are compared to simulated and experimental data of the irradiance fluctuations of a Gaussian beam wave propagating through the atmosphere along a horizontal path, near the ground, in the moderate-to-strong turbulence regime. Irradiance data were collected simultaneously at three receiving apertures of different sizes. Atmospheric parameters were inferred from the measurements and scintillation theory and were used to develop the parameters for the theoretical probability density functions. Numerical simulations were produced with the same $C_{n}^{2}$ value as the experimental data. Aperture-averaging effects were investigated by comparing the irradiance distributions for the three apertures at two different values of the structure parameter $C_{n}^{2}$, and, hence, different values of the coherence radius $\rho_{0}$. For the moderate-to-strong fluctuation regime, the GG distribution provides a good fit to the irradiance fluctuations collected by finite-sized apertures that are significantly smaller than $\rho_{0}$. For apertures larger than or equal to $\rho_{0}$, the irradiance fluctuations appear to be LN distributed. (C) 2007 Optical Society of America

OCIS codes: $\quad 010.1300,010.1330,290.5930$.
\end{abstract}

\section{Introduction}

The performance of a lasercom system operating in the atmosphere is reduced by optical turbulence, which causes irradiance fluctuations in the received signal (scintillation). The result is a randomly fading signal. Fade statistics for lasercom systems are determined from the probability density function (PDF) of the irradiance fluctuations. To determine the reliability of free-space optics systems, it is essential to have a mathematical model that accurately describes the PDF of the randomly fading irradiance signal. It is desirable to have a tractable, closed-form expression for the PDF that is valid under all conditions of irradiance fluctuations. In addition, the parameters of the PDF should depend directly on atmospheric parameters.

As early as the 1970s, it was widely accepted that the irradiance fluctuations obey lognormal (LN) sta-

F. S. Vetelino (fstromqu@ucf.edu), C. Young, and L. Andrews are with the Department of Mathematics, University of Central Florida, Orlando, Florida 32816, USA. J. Recolons is with the Department of Signal Theory and Communication, Universitat Politècnica de Catalunya, C/Sor Eulàlia d'Anzizu S/N, Campus Nord, 08034 Barcelona, Catalonia, Spain.

Received 21 September 2006; accepted 17 November 2006; posted 1 December 2006 (Doc. ID 75295); published 20 March 2007.

0003-6935/07/112099-10\$15.00/0

(C) 2007 Optical Society of America tistics in the weak fluctuation regime, ${ }^{1}$ but no PDF model existed for the moderate-to-strong fluctuation regime. ${ }^{1,2}$ It was later shown that a single family of density functions is inadequate to describe the irradiance fluctuations due to the nonstationary nature of atmospheric turbulence. Nonstationary scattering models were found to be governed by doubly stochastic processes, which are obtained by calculating the expected value of a conditional density function with a random, fluctuating mean. The mean of the conditional distribution is said to be smeared or modulated by the distribution of the mean. ${ }^{3,4}$

Several different modulations of two PDFs have been proposed as the distribution function for the irradiance fluctuations associated with a small-aperture receiver. In strong atmospheric turbulence, both the $\mathrm{K}$ distribution ${ }^{5-8}$ and the lognormally modulated exponential (LNME) distribution ${ }^{9}$ agree well with experimental data. The IK distribution ${ }^{3,10}$; the lognormally modulated Rician (LNMR) distribution, 1,2,11 also known as Beckmann's PDF12; and the gamma-gamma (GG) distribution $^{4,8,13}$ are candidates to describe the PDF of the irradiance fluctuations under all conditions of atmospheric turbulence. Both simulation ${ }^{12}$ and experimental ${ }^{14}$ data for spherical waves show strong support for the LNMR distribution in weak, moderate, and strong irradiance fluctuations. Also the GG distribution is supported by simulation data in all fluctuation regimes. ${ }^{13}$ 
The comparisons of theoretical PDFs with experimental or simulation data mentioned above are based on plane and spherical waves propagating along horizontal paths and use irradiance data collected by small (point) apertures. Some previous work exists that involves larger receiving apertures in the moderate-to-strong turbulence regime. ${ }^{9,15}$ They indicate that receiver-aperture-averaged irradiance is LN for sufficiently large apertures. As mentioned above, the LN distribution was believed to describe the irradiance fluctuations in weak turbulence. However, it was recently shown that irradiance fluctuations for plane and spherical waves propagating horizontally and collected by point apertures in the weak fluctuation regime are not truly LN.16,17 Experimental results, ${ }^{14}$ as well as simulation data, ${ }^{16,18}$ show deviations from LN statistics in the tails of the distribution. Theoretically, it has been shown that the PDF cannot be truly $\mathrm{LN}$ in the weak regime of turbulence by retaining perturbation terms up to second order in the Rytov approximation. ${ }^{17}$ Simulation data of Gaussian beam waves ${ }^{19,20}$ show that even the first-order term in the Rytov approximation has a nonlognormal behavior in the weak fluctuation regime when beam wander takes place. This occurs for focused beams along horizontal paths ${ }^{19}$ and collimated beams along ground-to-space paths. ${ }^{19,20}$

This work involves experimental data of a slightly diverged Gaussian beam wave propagating horizontally, near ground, in the moderate-to-strong regime of optical turbulence. Irradiance data were collected at three receiving apertures of different diameter. The path average values of important atmospheric parameters were inferred from the measurements and used to reproduce the experimental data with numerical simulations. The probability density of the irradiance fluctuations was calculated from both experimental data and simulation values for the three receiver apertures for both moderate and strong atmospheric turbulence. Comparisons were made with the LN and GG distributions. These distributions are attractive to work with because of their closed-form expressions and direct dependence on atmospheric parameters. Scintillation theory for the on-axis portion of a Gaussian beam wave was used in the calculations for the theoretical PDFs. However, for this particular experimental setup, spherical wave theory would give comparable results for the statistics of the laser beam.

\section{Aperture Averaging and Probability Density Function Models}

\section{A. Scale Sizes}

Scintillation, defined as the normalized irradiance fluctuations at the receiving aperture, is caused by a randomly changing refractive index along the propagation path. Pockets of air with constant index of refraction form turbulence cells called eddies, which range in scale size from the inner scale of turbulence $l_{0}$ to the large scale of turbulence $L_{0}$. Large-scale sizes have a refractive (focusing) effect on an optical wave, while small-scale sizes cause the wave to diffract. The refractive and diffractive scattering processes result in scintillation. In general, the diffractive small-scale contribution to scintillation is caused by scale sizes smaller than the Fresnel zone $(L / k)^{1 / 2}$ or the spatial coherence radius $\rho_{0}$, whichever is smallest, while the refractive large-scale effect is caused by scale sizes larger than the Fresnel zone or the scattering disk $L / k \rho_{0}$, whichever is largest. Consequently, in the moderate-to-strong fluctuation regime medium-sized eddies, smaller than the scattering disk and larger than the coherence radius, are ineffective in producing scintillation and will not contribute to the irradiance fluctuations. ${ }^{17}$

\section{B. Aperture Averaging}

If a receiving aperture is larger than a spatial scale size that produces the irradiance fluctuations, the receiver will average the fluctuations over the aperture and the scintillation will be less compared to scintillation measured with a point receiver. ${ }^{21}$ It has been shown that aperture averaging causes a shift of the relative spatial frequency content of the irradiance spectrum toward lower frequencies, since the fastest fluctuations caused by small-scale sizes average out. ${ }^{22}$ Hence, the scintillation measured by a receiving aperture is produced by scale sizes larger than the aperture.

In strong turbulence, there is a two-scale behavior in the irradiance flux variance obtained by a finitesize receiving aperture. A sharp decrease in scintillation is observed for increasing aperture sizes up to the coherence scale, $\rho_{0}$, after which there is a leveling effect, followed by a second decrease in the irradiance flux variance when the aperture exceeds the scattering disk $L / k \rho_{0} .{ }^{21,22}$ The leveling effect arises since medium-scale sizes are inefficient in producing scintillation in strong turbulence. Hence, if a receiving aperture is larger than $\rho_{0}$ in strong turbulence, the small-scale scintillation is mostly averaged out. This should affect the PDF of the irradiance fluctuations since existing models, developed for point receivers, are obtained by modulating the small- and largescale distributions. Intuitively, in strong turbulence, aperture averaging should shift the PDF toward the distribution of the large-scale fluctuations. The largescale fluctuations are assumed to be LN in both the LNMR and the LNME distributions, while it is assumed to be gamma distributed in the GG distribution.

\section{Lognormal Distribution}

The LN distribution is obtained by modeling the incoming field of an optical wave as a product of the scattered field from several independent scatterers. Taking the logarithm of the product and applying the central limit theorem result in a $\mathrm{LN}$ distributed field. ${ }^{2}$ Theoretically, the LN distribution follows from the Rytov approximation, which is valid under weak irradiance fluctuations and is based on a perturbation expansion in the exponent of the field. If only the 
first-order perturbation term is considered, the logarithm of the amplitude of the field can be expressed in terms of an integral over the refractive index fluctuations along the propagation path. The region of integration can be divided into numerous subregions, in which the refractive index fluctuations are uncorrelated. Therefore, by the central limit theorem, the logarithm of the amplitude is normally distributed. As a consequence, the logarithm of the irradiance is also normally distributed, and hence, the irradiance has a LN distribution. ${ }^{23}$ However, since the secondorder phase perturbation is not Gaussian, the irradiance fluctuations cannot be truly LN in the weak fluctuation regime. ${ }^{17}$

A LN distributed normalized irradiance $(\langle I\rangle=1)$ is described by ${ }^{17}$

$$
p_{I}(I)=\frac{1}{I \sqrt{2 \pi \sigma_{\ln I}^{2}}} \exp \left[-\frac{\left[\ln (I)+\frac{1}{2} \sigma_{\ln I}^{2}\right]^{2}}{2 \sigma_{\ln I}^{2}}\right], \quad I>0,
$$

where $\sigma_{\ln I}^{2}$ is the log-irradiance variance, which is related to the scintillation index $\sigma_{I}^{2}$ by the relation ${ }^{17}$

$$
\sigma_{\ln I}^{2}=\ln \left(\sigma_{I}^{2}+1\right),
$$

where $\ln$ is the natural logarithm. In weak turbulence $\sigma_{\ln I}^{2}$ is approximately equal to $\sigma_{I}^{2}$. In the moderate-to-strong fluctuation regime it is important to use the relation in Eq. (2).

\section{Gamma-Gamma Distribution}

The GG distribution for the irradiance is obtained by a modulation between the small-scale irradiance $y$ and the large-scale irradiance $x^{13,17}$ :

$$
I=x y,
$$

where $x$ and $y$ are assumed to be gamma distributed and statistically independent. A conditional PDF for the irradiance is given by the small-scale irradiance, which is assumed to have a fluctuating mean that is smeared, or modulated, by the distribution of the large-scale fluctuations. The unconditional PDF for the irradiance is a doubly stochastic process and is obtained by taking the expected value of the unconditional PDF, which results in the GG distribu$\operatorname{tion}^{13,17}$ :

$$
p_{I}(I)=\frac{2}{\Gamma(\alpha) \Gamma(\beta) I}(\alpha \beta I)^{(\alpha+\beta) / 2} K_{\alpha-\beta}(2 \sqrt{\alpha \beta I),} \quad I>0,
$$

where $I$ is the normalized irradiance, $\Gamma(x)$ is the gamma function, and $K_{\mu}(x)$ is the modified Bessel function of the second kind. $\alpha$ and $\beta$ are the parameters of the PDF, which represent the effective numbers of large- and small-scale scatterers, respectively. They are related to the scintillation index by calcu- lating the second moment of the GG distributed irradiance $^{13,17}$ :

$$
\left\langle I^{2}\right\rangle=\left(1+\frac{1}{\alpha}\right)\left(1+\frac{1}{\beta}\right)
$$

It follows from Eq. (3) that the second moment can also be written as

$$
\left\langle I^{2}\right\rangle=\left\langle x^{2}\right\rangle\left\langle y^{2}\right\rangle=\left(1+\sigma_{x}^{2}\right)\left(1+\sigma_{y}^{2}\right),
$$

where $\sigma_{x}^{2}$ and $\sigma_{y}^{2}$ are the large- and small-scale normalized variances (scintillation), respectively. Hence, the parameters of the GG distribution are identified as the inverse of the large- and small-scale scintillations:

$$
\begin{aligned}
& \alpha=\frac{1}{\sigma_{x}^{2}}=\frac{1}{\exp \left(\sigma_{\ln x}^{2}\right)-1}, \\
& \beta=\frac{1}{\sigma_{y}^{2}}=\frac{1}{\exp \left(\sigma_{\ln y}^{2}\right)-1} .
\end{aligned}
$$

Recently developed scintillation theory, ${ }^{17,22,24,25}$ valid in all regimes of turbulence, relates the small- and large-scale scintillations to the strength of turbulence and the inner and outer scales for various optical waves. Consequently, the parameters in the GG distribution are directly related to the atmospheric parameters $l_{0}, L_{0}$, and $C_{n}^{2}$. For a Gaussian beam wave, the relations are found in Ref. 17. The scintillation index, which is the parameter in the $\mathrm{LN}$ distribution, is related to the large- and small-scale log-irradiance variances, $\sigma_{\ln x}^{2}$ and $\sigma_{\ln y}^{2}$, respectively ${ }^{17}$ :

$$
\sigma_{I}^{2}=\exp \left(\sigma_{\ln x}^{2}+\sigma_{\ln y}^{2}\right)-1 .
$$

\section{Experiment and Simulation}

\section{A. Experimental Data}

The experiment was conducted during 7-11 February 2005 , at a laser range located near Adelaide, South Australia. The terrain was dry grassland. A continuous-wave laser operating at $1550 \mathrm{~nm}$ was launched horizontally into free space via a $45 \mathrm{~mm}$ diameter collimator, mounted $2.1 \mathrm{~m}$ above ground. The transmitted power was approximately $22 \mathrm{dBm}$ $(150 \mathrm{~mW})$ and the beam divergence was measured to be $0.46 \mathrm{mrad}$ (full angle). After propagating $1500 \mathrm{~m}$ along a horizontal path through the atmosphere, the beam was detected by three germanium photodiodes of 1,5 , and $13 \mathrm{~mm}$ diameter. No lenses were placed in front of the detectors.

The detectors were mounted on a tripod, positioned $2.2 \mathrm{~m}$ above ground, and the signal was captured with a sampling rate of $10 \mathrm{kHz}$. Great caution was taken to avoid clipping in the received irradiance data. Data were taken in 5 min intervals, and the background radiation was measured before and after each run. 
During the data collection, the weather ranged from full sunshine to mostly sunny, with air temperatures in the range of $18-27{ }^{\circ} \mathrm{C}$ and wind speeds between 0 and $7 \mathrm{~m} / \mathrm{s}$ (cross wind speed of $0-5 \mathrm{~m} / \mathrm{s}$ ). A more detailed description of the experiment is found in Ref. 26.

The probability density of the natural logarithm of the normalized irradiance was determined for each receiver aperture for 5 min intervals of collected data. To obtain the true experimental irradiance, the background noise was subtracted from each data point. The background noise was estimated to be the mean value of the background measurement taken before and after each 5 min interval of collected data. The normalized irradiance was obtained by dividing each corrected data point by the $5 \mathrm{~min}$ average of the corrected data. To capture the behavior in the tails of the PDF, the natural logarithm of the normalized irradiance, referred to as the normalized log irradiance, was calculated and used to sort the data into histogram bins. For values of the normalized log irradiance between -5 and -2 , the bin width was 1 . For values from -2 to 2 , and from 2 to 5 , the corresponding bin widths were 0.2 and 0.5 . The different bin widths were used to avoid empty bins. The number of data points in each bin was calculated for the 5 min time interval. The probability density was obtained by dividing the number of data points in each bin by the bin width and the total number of data points in the time interval $\left(3 \times 10^{6}\right)$. The midpoint of the normalized log irradiance in each bin was used as the corresponding log-irradiance value for the experimental probability density when plotting the PDF.

To compare the PDF of the experimental data with theory and simulation data, the atmospheric conditions along the propagation path are required. Optical measurements, in combination with a scintillation theory developed by Andrews et al. ${ }^{17,25}$ were used to infer the refractive index structure parameter $C_{n}^{2}$, the inner scale of turbulence $l_{0}$, and the outer scale of turbulence $L_{0}$, along the propagation path. ${ }^{26}$ The theoretical expressions for the scintillation index, at a fixed range and wavelength, depend on four parameters: $C_{n}^{2}, l_{0}, L_{0}$, and $D$. By specifying three different collecting aperture sizes, and measuring the corresponding scintillation index experimentally, a minimization problem for the atmospheric parameters was created. It was solved numerically with the downhill simplex method, ${ }^{26,27}$ which is a multidimensional minimization algorithm that requires only function evaluations, not derivatives. The experimental scintillation index was measured for the same 5 min of data used to calculate the PDF, at each receiving aperture (1, 5 , and $13 \mathrm{~mm}$ diameter).

Two sets of 5 min intervals of experimental data, collected at 8:47 a.m.-8:52 a.m. and 2:16 p.m.-2:21 p.m. on 11 February 2005, are presented in this paper. The inferred atmospheric parameters for the first data set were $C_{n}^{2}=6.5 \times 10^{-14}, l_{0}=6.1 \mathrm{~mm}$, and $L_{0}=1.3 \mathrm{~m}$, and for the second data set $C_{n}^{2}=4.6$ $\times 10^{-13}, l_{0}=4.6 \mathrm{~mm}$, and $L_{0}=1.1 \mathrm{~m}$. These values were used to produce simulation data and to calculate parameters for the theoretical PDFs.

The LN and GG distributions for the log irradiance are obtained by the following transformation of variables:

$$
p_{z}(z)=\left.p_{I}(I) \frac{d I}{d z}\right|_{I=e^{z}}=e^{z} p_{I}\left(e^{z}\right),
$$

where $z=\ln I$ is the normalized log irradiance. The $\alpha$ and $\beta$ parameters in the GG distribution and $\sigma_{\ln I}^{2}$ in the $\mathrm{LN}$ distribution were calculated from the inferred $C_{n}^{2}, l_{0}$, and $L_{0}$ values by using expressions from the scintillation theory for a Gaussian beam wave presented in Refs. 17 and 26.

\section{B. Simulation}

To reproduce the experimental results, a numerical simulation of the propagation of a Gaussian beam in atmospheric conditions was performed using a wave-optics code. The wave-optics code is based on a split-step technique, in which the light waves are propagated in the transformed domain and the effect of atmospheric turbulence along the propagation path is simulated by a series of uncorrelated random phase screens. These phase screens are generated under the Markov approximation in the spectral domain by generating a set of Gaussian distributed random numbers with zero mean and variances given by

$$
\left\langle\left|A_{\mu \nu}\right|^{2}\right\rangle=2 \pi k^{2} \Delta z \Phi_{n}\left(\boldsymbol{\kappa}_{\mu \nu}\right)(\Delta \kappa)^{2},
$$

where $A_{\mu \nu}$ are the random harmonic amplitudes associated with the transverse wave vectors $\boldsymbol{\kappa}_{\mu v}, k$ is the propagating field wavenumber, $\Delta z$ is the thickness of the phase screen along the propagation path, $\Delta \kappa$ is the grid spacing in the transverse frequency domain, and $\Phi_{n}\left(\boldsymbol{\kappa}_{\mu v}\right)$ is the spectral power density of the refractive index fluctuations. Kolmogorov's spectrum was used and inner- and outer-scale effects ignored. By applying the Fourier transform to the random amplitudes $A_{\mu \nu}$, the random phase fluctuations $\Delta \theta_{l m}$ are generated over an $N \times N$ grid. This procedure is repeated at each phase screen along the path. To overcome the problem of undersampling at the lowest spectral frequencies, a subharmonics technique was used with a spectral correction in the subharmonic regime. ${ }^{28,29}$

The simulated beam was a diverging Gaussian beam focused at a distance $F_{0}=-69.9 \mathrm{~m}$ (calculated from the experimental divergence angle of $0.46 \mathrm{mrad}$ ) behind the transmitter and with a transmitter beam radius of $W_{0}=1.59 \mathrm{~cm} . W_{0}$ is defined as the radius at which the irradiance is $e^{-2}$ of its on-axis value and fulfills the ratio $W_{0}=\sqrt{8} D$, where $D=4.5 \mathrm{~cm}$ is the beam hard aperture diameter at the transmitter. The beam was propagated along a $1.5 \mathrm{~km}$ horizontal path, which was divided into 15 equivalent phase screens, each covering a distance $z=100 \mathrm{~m}$. The free-space wavelength was $\lambda=1.55 \mu \mathrm{m}$, and the turbulence strength was either $C_{n}^{2}=6.47 \times 10^{-14}$ or $C_{n}^{2}=4.58$ 
$\times 10^{-13}$, corresponding to the two experimental cases. Each phase screen included a total number of 700 grid points along each dimension, separated by a $1.573 \mathrm{~mm}$ grid spacing, thus making the total screen width $1.1 \mathrm{~m}$ in both transverse dimensions. The two larger receiver apertures (5 and $13 \mathrm{~mm}$ ) used in the experiment were simulated by averaging the irradiance values at the receiver plane over a circular area with the same aperture diameter, centered at the optical axis. The $1 \mathrm{~mm}$ aperture was compared with the irradiance values measured at a single pixel lo- cated at the optical axis. The pixel square area is $2.47 \mathrm{~mm}^{2}$ in the simulations, whereas the circular area of the $1 \mathrm{~mm}$ aperture is smaller by a factor of $\pi$. Hence, a circular receiver aperture of diameter $\pi^{1 / 2} \approx 1.8 \mathrm{~mm}$ has the same area as the pixel. To simulate the PDF of the irradiance fluctuations, a total number of 40,000 irradiance values were obtained for each different case. The probability density of the simulated values of the normalized log irradiance was obtained in a similar way as described for the experimental data.

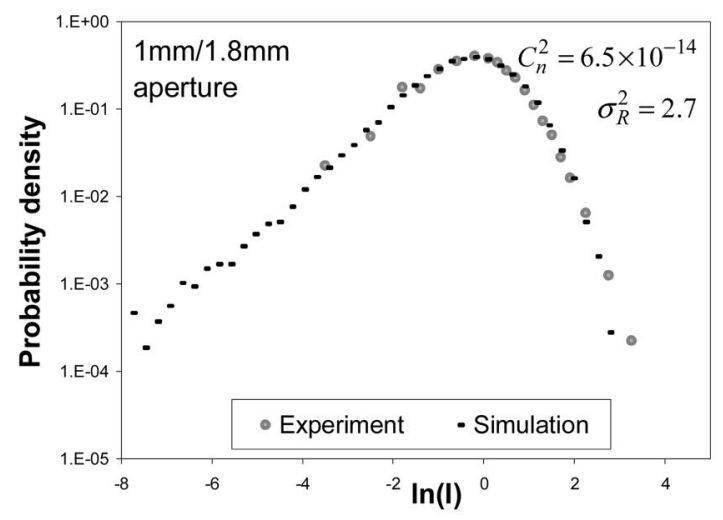

(a)

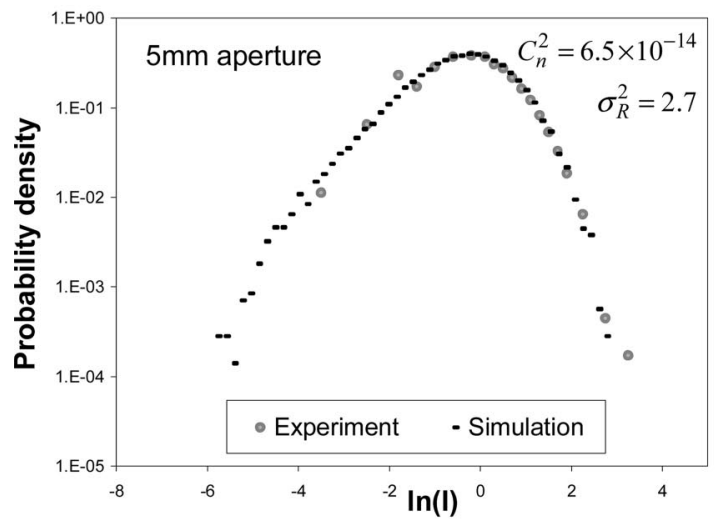

(c)

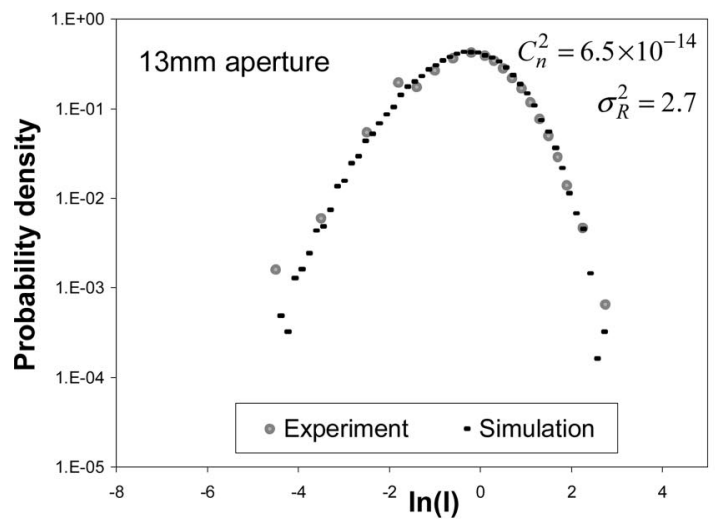

(e)

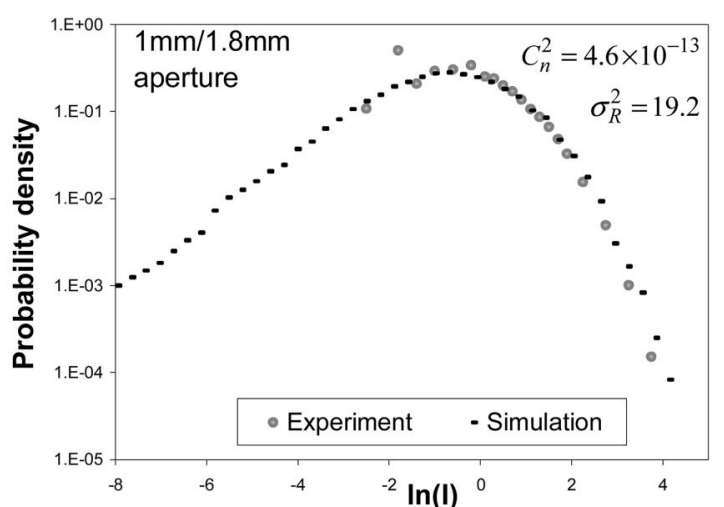

(b)

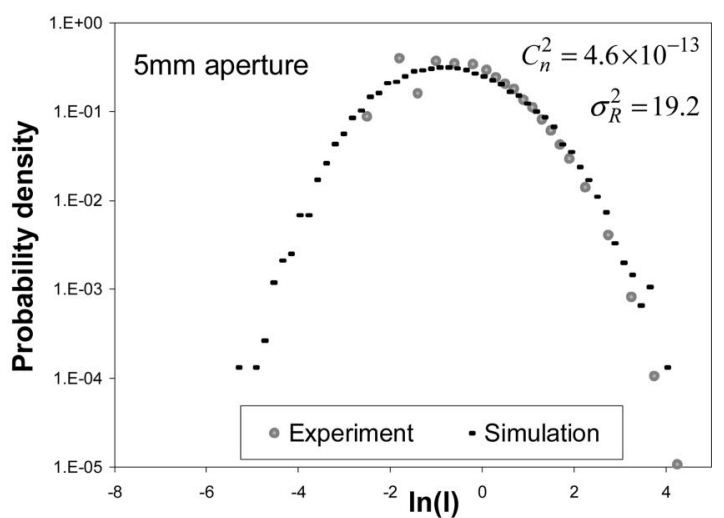

(d)

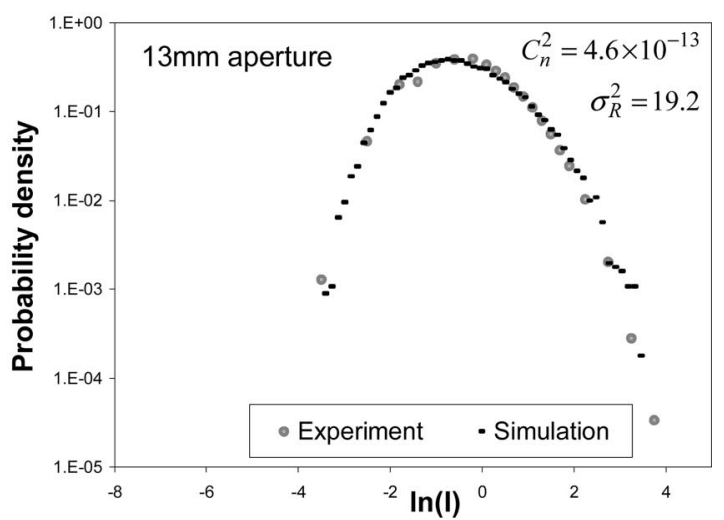

(d)

Fig. 1. PDF of the normalized log irradiance, $\ln (I)$, based on the experimental data (circles) and the simulation data (crosses) for the receiving apertures of diameter (a), (b) $1 \mathrm{~mm}$, (c), (d) $5 \mathrm{~mm}$, and (e), (f) $13 \mathrm{~mm}$. Two cases of turbulence strength are shown: (a), (c), (e) $C_{n}^{2}=6.47 \times 10^{-14}$ and (b), (d), (f) $C_{n}^{2}=4.58 \times 10^{-13}$. 
Since the simulation data were obtained using the Kolmogorov spectrum, the parameters of the theoretical PDFs could be calculated by ignoring inner- and outer-scale effects in the theoretical expressions for the log-irradiance variance, $\sigma_{\ln I}^{2}$, and the $\alpha$ and $\beta$ parameters. However, the scintillation index calculated directly from the simulation data does not match predictions made by the scintillation theory. Hence, the $\sigma_{\ln I}^{2}$ parameter in the LN distribution was determined by using Eq. (2) with the scintillation index calculated directly from the simulation data. The $\alpha$ and $\beta$ parameters in the GG distribution were obtained by doing a best fit to the simulation data, while maintaining the relationship

$$
\sigma_{I}^{2}=\left(1+\frac{1}{\alpha}\right)\left(1+\frac{1}{\beta}\right)-1,
$$

where the scintillation index $\sigma_{I}^{2}$ was calculated directly from the simulation data.

\section{Results}

A comparison between the experimental and simulation data for all three receiving aperture sizes and the two cases of turbulence strength $\left(C_{n}^{2}=6.47 \times 10^{-14}\right.$ and $C_{n}^{2}=4.58 \times 10^{-13}$ ) is shown in Fig. 1. Each subplot shows the results from one receiving aperture and one turbulence condition. The circles show the probability density function for the normalized log irradiance $\ln (I)$ obtained from the experimental data. The values of $\ln (I)$ corresponding to each circle are the midpoints of each histogram bin. The crosses display the PDF determined from the simulation values. In all cases, the experimental and simulation data have a close fit.

The parameter values used to determine the theoretical PDFs for $\ln (I)$ are displayed in Table 1 . For the experimental data, the parameter values were calculated using scintillation theory and atmospheric parameters $\left(C_{n}^{2}, l_{0}\right.$, and $\left.L_{0}\right)$ inferred from the two different cases of experimental data. The inferred atmospheric parameters are also shown in Table 1. The Rytov variance $\sigma_{R}^{2}=1.23 C_{n}^{2} k^{7 / 6} L^{11 / 6}$, where $k$ is the wavenumber and $L$ is the propagation distance, is provided since it is a commonly used turbulence parameter. The parameter values for the theoretical PDFs for the simulation data were obtained by calculating $\sigma_{I}^{2}$ directly from the simulation data and by doing a best fit for the $\alpha$ and $\beta$ parameters. The $\alpha$ and $\beta$ parameters were used to obtain the GG distribution. As shown in Table 1, the weak turbulence approximation $\sigma_{I}^{2} \approx \sigma_{\ln I}^{2}$ does not hold. Hence, it is important to use the $\sigma_{\ln I}^{2}$ value when calculating the LN PDF.

Comparisons between the theoretical PDFs and the experimental and simulation data are displayed in Figs. 2 and 3, respectively. The solid curve represents the GG distribution, while the dashed curve is the LN distribution. Each subplot shows the results from one receiving aperture and one turbulence condition. The comparisons with the theoretical PDFs are focused on the tail of the PDF for small irradiance values, since that is the important region of the PDF when predicting fade statistics for lasercom systems. The comparisons between data and theory are significantly different for the two different turbulence conditions and receiving apertures of different sizes. As shown in Figs. 2 and 3, both the simulation and experimental data for $C_{n}^{2}=6.47 \times 10^{-14}$ have a close fit to the GG distribution for both the 1 and $5 \mathrm{~mm}$ receiving apertures. For $C_{n}^{2}=6.47 \times 10^{-14}$ and the $13 \mathrm{~mm}$ aperture, the experimental data support a GG distribution, while the simulation data lie between the GG and LN distributions. For $C_{n}^{2}=4.58 \times$ $10^{-13}$, the simulation data for the $1 \mathrm{~mm}$ receiving aperture fall between the GG and LN distributions, while the simulation data for the $5 \mathrm{~mm}$ aperture have a close fit to the LN distribution. For the two smaller apertures, the resolution of the experimental results in the tail of the PDF for small irradiance values is not good enough to draw any conclusions. For $C_{n}^{2}=4.58 \times 10^{-13}$ and the $13 \mathrm{~mm}$ aperture, the

Table 1. Atmospheric Parameters Inferred from the Experimental Data and the PDF Parameters Used to Calculate the LN and GG Distributions when Compared to the Experimental Data and the Simulation Data

\begin{tabular}{|c|c|c|c|c|c|c|c|c|}
\hline \multirow[b]{2}{*}{$\begin{array}{l}\text { Atmospheric } \\
\text { parameter }\end{array}$} & \multicolumn{4}{|c|}{ Case I } & \multicolumn{4}{|c|}{ Case II } \\
\hline & $\begin{array}{c}C_{n}^{2} \\
\left(\mathrm{~m}^{-2 / 3}\right) \\
6.5 \times 10^{-14}\end{array}$ & $\begin{array}{c}l_{0} \\
(\mathrm{~mm}) \\
6.1\end{array}$ & $\begin{array}{c}L_{0} \\
(\mathrm{~m}) \\
1.3\end{array}$ & 2.7 & $\begin{array}{c}C_{n}^{2} \\
\left(\mathrm{~m}^{-2 / 3}\right) \\
4.6 \times 10^{-13}\end{array}$ & $\begin{array}{c}l_{0} \\
(\mathrm{~mm}) \\
4.6\end{array}$ & $\begin{array}{c}L_{0} \\
(\mathrm{~m}) \\
1.1\end{array}$ & $\sigma_{R}^{2}$ \\
\hline & \multicolumn{4}{|c|}{ Experimental Data } & \multicolumn{4}{|c|}{ Experimental Data } \\
\hline PDF parameter & $\alpha$ & $\beta$ & $\sigma_{I}^{2}$ & $\sigma_{\ln I}^{2}$ & $\alpha$ & $\beta$ & $\sigma_{I}^{2}$ & $\sigma_{\ln I}^{2}$ \\
\hline $1 \mathrm{~mm}$ aperture & 1.87 & 2.07 & 1.28 & 0.83 & 1.09 & 1.11 & 2.64 & 1.29 \\
\hline $5 \mathrm{~mm}$ aperture & 1.89 & 2.12 & 1.25 & 0.81 & 1.10 & 1.31 & 2.38 & 1.22 \\
\hline \multirow[t]{2}{*}{$13 \mathrm{~mm}$ aperture } & 2.05 & 2.45 & 1.10 & 0.74 & 1.13 & 2.47 & 1.65 & 0.97 \\
\hline & \multicolumn{4}{|c|}{ Simulation Data } & \multicolumn{4}{|c|}{ Simulation Data } \\
\hline PDF parameter & $\alpha$ & $\beta$ & $\sigma_{I}^{2}$ & $\sigma_{\ln I}^{2}$ & $\alpha$ & $\beta$ & $\sigma_{I}^{2}$ & $\sigma_{\ln I}^{2}$ \\
\hline $1.8 \mathrm{~mm}$ aperture & 1.49 & 3.00 & 1.23 & 0.80 & 0.88 & 0.88 & 3.55 & 1.51 \\
\hline $5 \mathrm{~mm}$ aperture & 1.83 & 2.42 & 1.19 & 0.78 & 0.96 & 0.96 & 3.15 & 1.42 \\
\hline $13 \mathrm{~mm}$ aperture & 2.38 & 2.38 & 1.01 & 0.70 & 1.28 & 1.28 & 2.16 & 1.15 \\
\hline
\end{tabular}




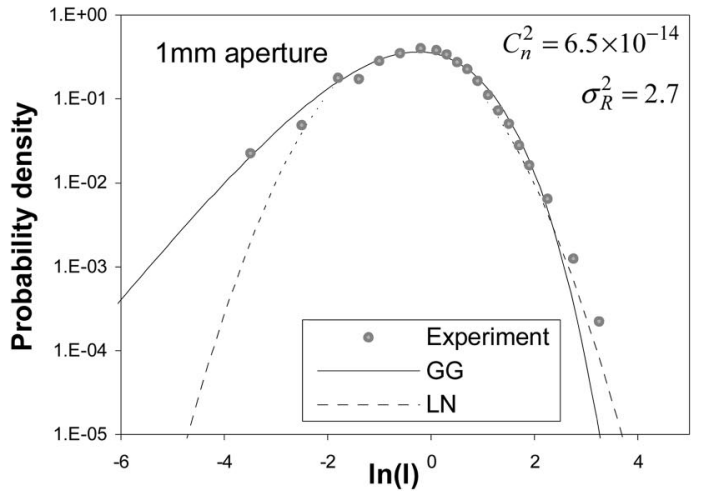

(a)

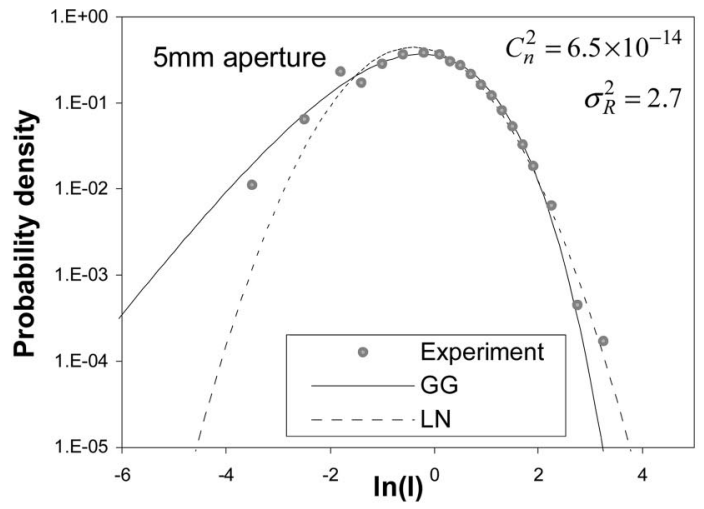

(c)

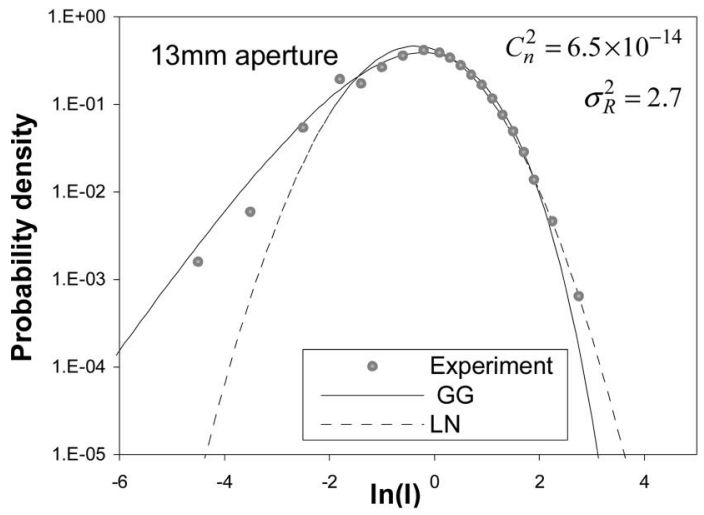

(e)

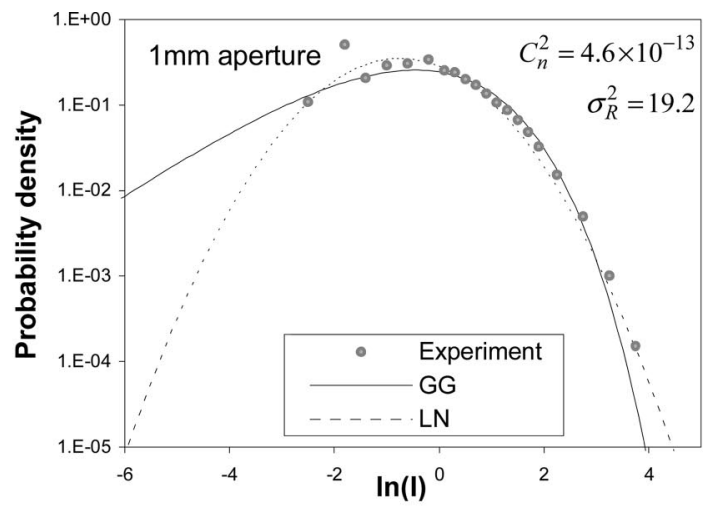

(b)

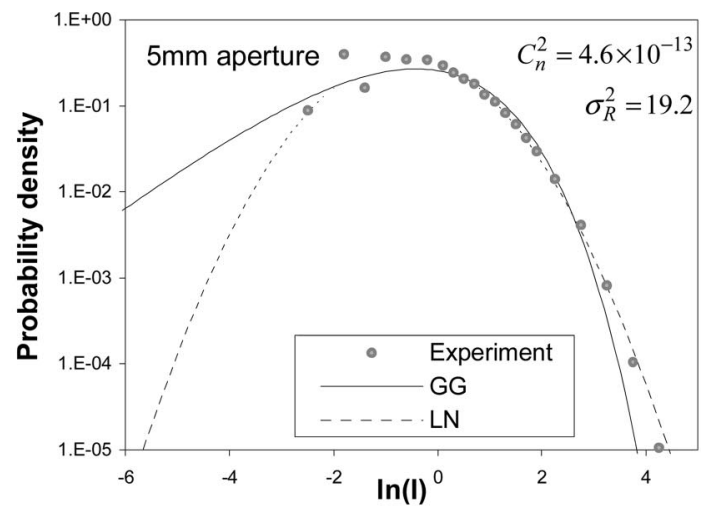

(d)

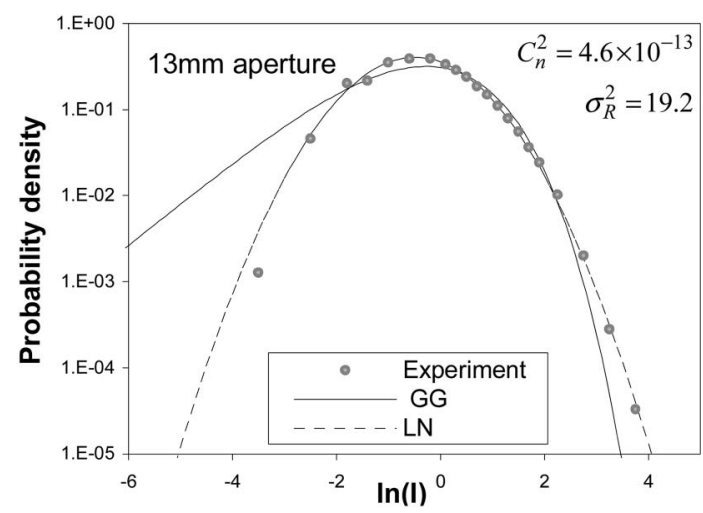

(f)

Fig. 2. PDF of the normalized $\log$ irradiance $\ln (I)$ for the receiving apertures of diameter (a), (b) $1 \mathrm{~mm}$, (c), (d) $5 \mathrm{~mm}$, and (e), (f) $13 \mathrm{~mm}$. Comparisons are made between the experimental data and the GG distribution and the LN distribution for (a), (c), (e) $C_{n}^{2}=6.47$ $\times 10^{-14}$ and (b), (d), (f) $C_{n}^{2}=4.58 \times 10^{-13}$.

experimental data support the $\mathrm{LN}$ distribution and the simulation data are closer to the LN distribution than the GG distribution. The comparisons between data and theory are summarized in Table 2.

Figure 4 shows a graph of the Fresnel zone $(L / k)^{1 / 2}$, the scattering disk $L / k \rho_{0}$, and the coherence radius $\rho_{0}$ as a function of turbulence strength. The two $C_{n}^{2}$ values corresponding to the different cases of the experimental and simulation data are marked with vertical lines. The dashed horizontal lines represent the diameter of the three receiving apertures. Figure 4 shows that the first case with $C_{n}^{2}=6.47 \times 10^{-14}$ takes place in the onset of strong turbulence, since it occurs when the Fresnel zone, scattering disk, and the coherence radius are of equal size. Everything to the right of the interception is considered strong turbulence. Hence, the second case with $C_{n}^{2}=4.58 \times$ $10^{-13}$ takes place in the regime of strong atmospheric turbulence. Note that the size of the receiving apertures relative to the coherence radius is different for the two turbulence cases. All aperture diameters are smaller than the coherence radius for $C_{n}^{2}=6.47$ $\times 10^{-14}$, but only the $1 \mathrm{~mm}$ aperture is significantly smaller for $C_{n}^{2}=4.58 \times 10^{-13}$. The labels GG and LN 


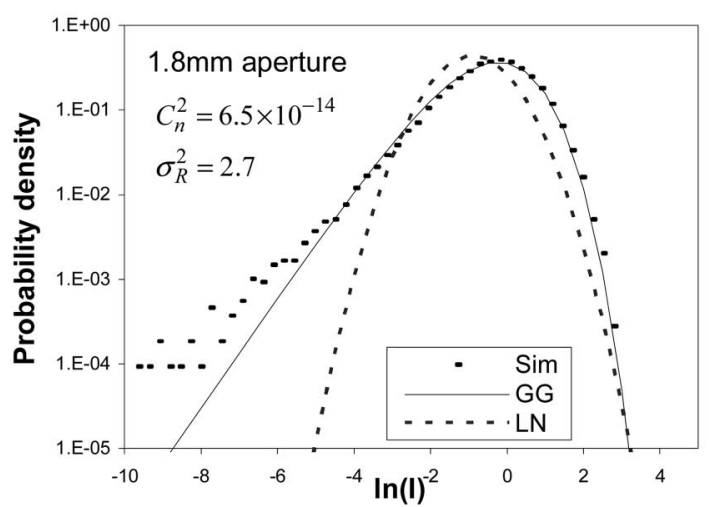

(a)

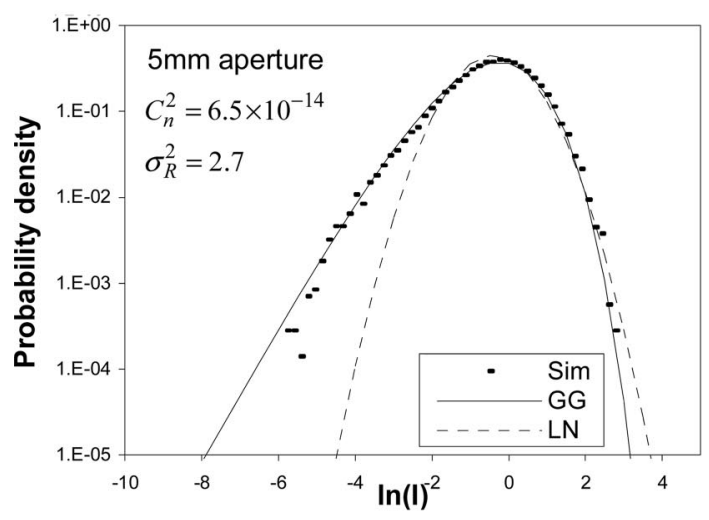

(c)

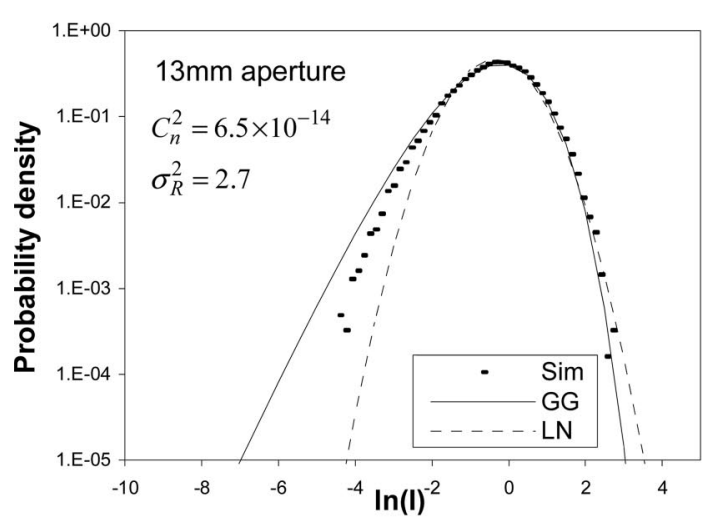

(e)

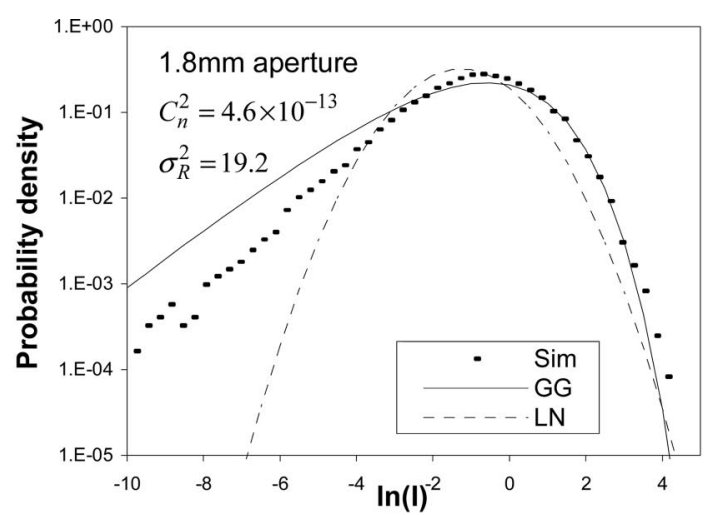

(b)

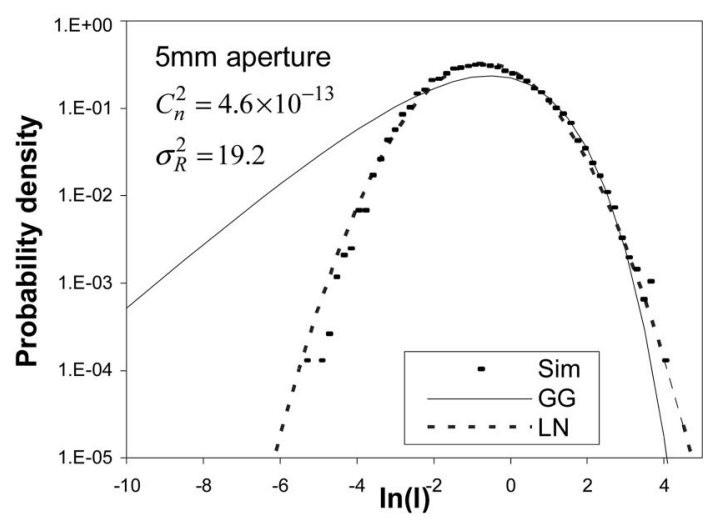

(d)

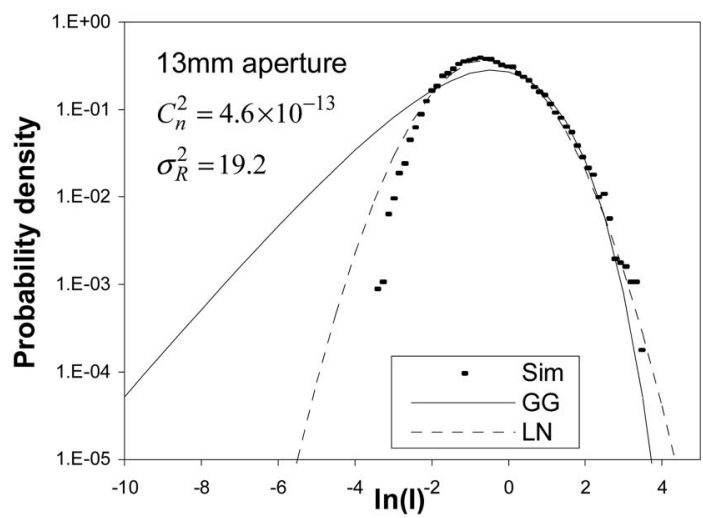

(f)

Fig. 3. PDF of the normalized $\log$ irradiance $\ln (I)$ for the receiving apertures of diameter (a), (b) $1 \mathrm{~mm}$, (c), (d) $5 \mathrm{~mm}$, and (e), (f) $13 \mathrm{~mm}$. Comparisons are made between the simulation data and the GG distribution and the LN distribution for (a), (c), (e) $C_{n}^{2}=6.47 \times 10^{-14}$ and (b), (d), (f) $C_{n}^{2}=4.58 \times 10^{-13}$.

correspond to where the data support the GG and the LN PDFs, respectively, while GG/LN denotes where the data lie between the two distributions.

\section{Discussion}

The shift from a GG distribution to a LN distribution for the $5 \mathrm{~mm}$ aperture as the strength of turbulence increases is clearly seen in Figs. 3(c) and 3(d). As seen in Fig. 4, in the case of the GG distribution, the $5 \mathrm{~mm}$ aperture is significantly smaller than the coherence radius, but the two quantities are of similar size in the case of the LN distribution. Also the $13 \mathrm{~mm}$ ap- erture irradiance has a $\mathrm{LN}$ distribution when it is larger than the coherence radius in strong turbulence. The comparison between the receiver-aperture size and the coherence radius is of interest because scale sizes larger than the coherence radius and smaller than the scattering disk are ineffective in producing scintillation in the moderate-to-strong fluctuation regime. Hence, all small-scale scintillation is produced by scale sizes smaller than the coherence radius and their contribution to the irradiance fluctuations is averaged out when the receiving aperture is larger than or similar in size to the coherence radius. 
Table 2. PDF of the Irradiance Fluctuations for the Three Receiver Aperture Diameters and the Two Different Cases of Turbulence Based on the Experimental Data and Simulation Data, Respectively ${ }^{a}$

\begin{tabular}{|c|c|c|c|c|}
\hline \multicolumn{2}{|c|}{$\begin{array}{c}\mathrm{I}: C_{n}^{2}=6.47 \times 10^{-14} \\
\rho_{0}=20 \mathrm{~mm}\end{array}$} & \multicolumn{3}{|c|}{$\begin{array}{c}\text { II: } C_{n}^{2}=4.58 \times 10^{-13} \\
\rho_{0}=6.2 \mathrm{~mm}\end{array}$} \\
\hline \multicolumn{5}{|c|}{ Experimental Data } \\
\hline Aperture diameter & GG LN & Aperture diameter & GG & $\mathrm{LN}$ \\
\hline $1 \mathrm{~mm}$ & $\mathrm{X}$ & $1 \mathrm{~mm}$ & Inconc & usive \\
\hline $5 \mathrm{~mm}$ & $\mathrm{X}$ & $5 \mathrm{~mm}$ & Inconc & usive \\
\hline $13 \mathrm{~mm}$ & $\mathrm{X}$ & $13 \mathrm{~mm}$ & & $\mathrm{X}$ \\
\hline \multicolumn{5}{|c|}{ Simulation Data } \\
\hline Aperture diameter & GG LN & Aperture diameter & GG & $\mathrm{LN}$ \\
\hline $1.8 \mathrm{~mm}$ & $\mathrm{X}$ & $1.8 \mathrm{~mm}$ & & $x$ \\
\hline $5 \mathrm{~mm}$ & $\mathrm{X}$ & $5 \mathrm{~mm}$ & & $\mathrm{X}$ \\
\hline $13 \mathrm{~mm}$ & $\mathrm{X}$ & $13 \mathrm{~mm}$ & & $\mathrm{X}(?)$ \\
\hline
\end{tabular}

${ }^{a} \rho_{0}$ is the coherence radius.

Consequently, under these conditions, the doubly stochastic process that describes the irradiance fluctuations for a point receiver goes into a single distribution - the PDF of the large-scale fluctuations. These results indicate that the large-scale fluctuation is LN, which is in agreement with the assumptions made for the LNMR (Ref. 11) and LNME (Ref. 9) distributions.

The doubly stochastic PDF, developed for a point receiver, accurately describes the irradiance fluctuations collected from apertures significantly smaller than the coherence radius in the moderate-to-strong turbulence regime. With aperture diameters slightly smaller than the coherence radius, portions of the small-scale scintillation are still modulating the large-scale fluctuations, which result in deviations from both the LN distribution and the doubly stochastic process. Based on previous results from a spherical wave, ${ }^{9}$ it is assumed that the irradiance fluctuations will be LN in the weak regime of turbulence if the collecting aperture is larger than or equal to the Fresnel zone.

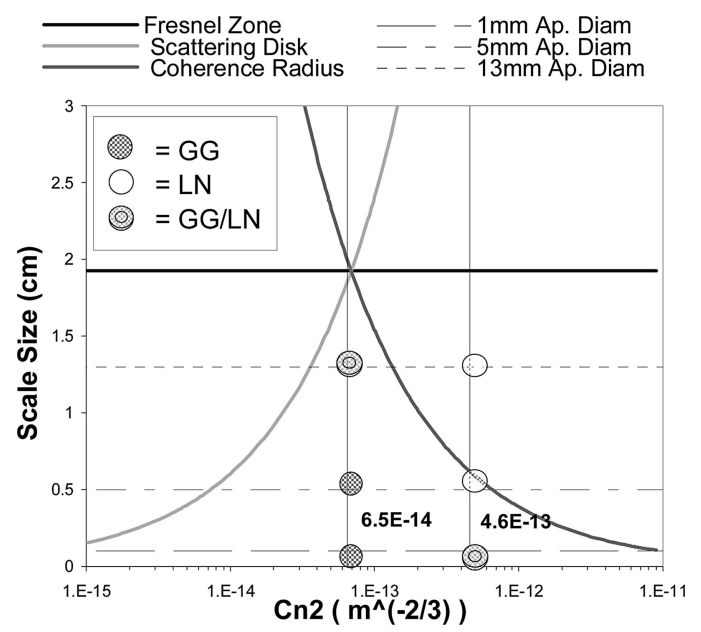

Fig. 4. Three receiver-aperture diameters (Ap. Diam) compared to the Fresnel zone, scattering disk and coherence radius for different values of $C_{n}^{2}$.
A gamma distribution was unsuccessfully fitted to the data in Figs. 2(f), 3(d), and 3(f) (the results are not shown in the graphs). Hence, the large-scale fluctuations do not appear to be gamma distributed, as is assumed in the GG distribution. However, the GG distribution agrees well with the data for receiver apertures that are significantly smaller than the coherence radius in the moderate-to-strong fluctuation regime. The closed-form GG distribution is thus an attractive alternative to the more complicated LNMR distribution, which has an integral representation with poor convergence properties. In fact, the gamma distribution can approximate both the LN and the Rician distributions, which are the modulated densities in the LNMR PDF. Hence, the GG distribution can be seen as a tractable, closed-form approximation to the LNMR PDF.13

As shown in Fig. 1, the PDF obtained from the experimental data agrees well with the PDF corresponding to the simulation values for all three receiveraperture sizes and both cases of turbulence strength. This confirms that the method used to infer atmospheric parameters from the experimental data yields reasonable $C_{n}^{2}$ values, since the simulation data were produced with the inferred $C_{n}^{2}$ values. Because the numerical simulation ignores inner- and outerscale effects, no conclusions can be made about the accuracy of the inferred inner- and outer-scale values.

Another observation made in Fig. 1 is that the simulation results yield lower probability levels for low irradiance values than the experimental data do, even though the simulation results are based on 40,000 irradiance readings, while the experimental results used three million data points. When collecting the experimental data, linear amplifiers were used and great caution was made to avoid clipping of large peaks in the irradiance, since clipping would skew the PDF. As a result, the resolution for small irradiance readings was limited. The results are believed to improve if logarithmic amplifiers are used. However, the main reason for the lack of low probability levels for small experimental irradiance values is most likely due to background noise. To remove the impact of the background noise on the PDF, the mean value of the background measurement taken before and after each 5 min interval of collected data was subtracted from each data point. Nevertheless, as seen in Figs. 1(e) and 1(f), the experimental data reach the same probability level for small irradiance values $\left(10^{-3}\right)$ as the simulation data.

This analysis was made under the assumption of no beam wander effects. The assumption was confirmed by the simulation data, which indicated that no beam wander took place.

\section{Conclusions}

For the moderate-to-strong fluctuation regime, the GG distribution provides a good fit to the irradiance fluctuations collected by finite-sized apertures that are significantly smaller than the coherence radius $\rho_{0}$. For apertures larger than or equal to the coher- 
ence radius, the irradiance fluctuations are LN distributed. The doubly stochastic distribution, obtained by modulating the small- and large-scale scintillations and developed for point receivers, will go into a single distribution describing the large-scale fluctuations as the receiving aperture exceeds the coherence radius and small-scale fluctuations are reduced by aperture averaging. Consequently, the large-scale scintillation appears to be LN.

\section{References}

1. J. W. Strohbehn, T.-I. Wang, and J. P. Speck, "On the probability density distribution of line-of-sight fluctuations of optical signals," Radio Sci. 10, 59-70 (1975).

2. J. W. Strohbehn, "Modern theories in the propagation of optical waves in a turbulent medium," in Laser Beam Propagation in the Atmosphere, J. W. Strohbehn, ed. (Springer-Verlag, 1978), pp. 97-104.

3. L. C. Andrews and R. L. Phillips, "I-K-distribution as a universal propagation model of laser beams in atmospheric turbulence,” J. Opt. Soc. Am. A 2, 160-163 (1985).

4. D. J. Lewinski, "Nonstationary probabilistic target and clutter scattering models," IEEE Trans. Antennas Propag. AP-31, 490-498 (1983).

5. E. Jakeman and P. N. Pusey, "The significance of $K$ distributions in scattering experiments," Phys. Rev. Lett. 40, 546-550 (1978).

6. G. Parry and P. N. Pusey, " $K$ distributions in atmospheric propagation of laser light," J. Opt. Soc. Am. 69, 796-798 (1979).

7. E. Jakeman, "On the statistics of $K$-distributed noise," J. Phys. A 13, 31-48 (1980).

8. M. C. Teich and P. Diament, "Multiply stochastic representations for $K$ distributions and their Poisson transforms," J. Opt. Soc. Am. A 6, 80-91 (1989).

9. J. H. Churnside and R. J. Hill, "Probability density of irradiance scintillations for strong path-integrated refractive turbulence," J. Opt. Soc. Am. A 4, 727-733 (1987).

10. L. C. Andrews and R. L. Phillips, "Mathematical genesis of the $I$-K-distribution for random optical fields," J. Opt. Soc. Am. A 3, 1912-1919 (1986).

11. J. H. Churnside and S. F. Clifford, "Log-normal Rician probability-density function of optical scintillations in the turbulent atmosphere," J. Opt. Soc. Am. A 4, 1923-1930 (1987).

12. R. J. Hill and R. G. Frehlich, "Probability distribution of irradiance for the onset of strong scintillation," J. Opt. Soc. Am. A 14, 1530-1540 (1997).

13. M. A. Al-Habash, L. C. Andrews, and R. L. Phillips, "Mathematical model for the irradiance PDF of a laser beam propagating through turbulent media," Opt. Eng. 40, 1554-1562 (2001).
14. J. H. Churnside and R. G. Frehlich, "Experimental evaluation of log-normally modulated Rician and $I K$ models of optical scintillation in the atmosphere," J. Opt. Soc. Am. A 6, 17601766 (1989).

15. Z. Azar, H. M. Loebenstein, G. Appelbaum, E. Azoulay, U. Halavee, M. Tamir, and M. Tur, "Aperture averaging of the two-wavelength intensity covariance function in atmospheric turbulence," Appl. Opt. 24, 2401-2407 (1985).

16. A. D. Wheelon, "Skewed distribution of irradiance predicted by second-order Rytov approximation," J. Opt. Soc. Am. A 18, 2789-2798 (2001).

17. L. C. Andrews, R. L. Phillips, and C. Y. Hopen, Laser Beam Scintillation with Applications (SPIE Optical Engineering Press, 2001).

18. S. M. Flatté, C. Bracher, and G.-Yu Wang, "Probability-density functions of irradiance for waves in atmospheric turbulence calculated by numerical simulations," J. Opt. Soc. Am. A 11, 2080-2092 (1994).

19. G. J. Baker, "Gaussian beam weak scintillation: low-order turbulence effects and applicability of the Rytov method," J. Opt. Soc. Am. A 23, 395-417 (2006).

20. F. Dios, J. A. Rubio, A. Rodríguez, and A. Comerón, "Scintillation and beam-wander analysis in an optical ground stationsatellite uplink," Appl. Opt. 43, 3866-3873 (2004).

21. J. H. Churnside, "Aperture averaging of optical scintillation in the turbulent atmosphere," Appl. Opt. 30, 1982-1994 (1991).

22. L. C. Andrews, R. L. Phillips, and C. Y. Hopen, "Aperture averaging of optical scintillations: power fluctuations and temporal spectrum," Waves Random Media 10, 53-70 (2000).

23. V. I. Tatarskii, The Effects of Turbulent Atmosphere on Wave Propagation (National Technical Information Office, 1971), p. 292.

24. L. C. Andrews, R. L. Phillips, C. Y. Hopen, and M. A. AlHabash, "Theory of optical scintillation," J. Opt. Soc. Am. A 16, 1417-1429 (1999).

25. L. C. Andrews, M. A. Al-Habash, C. Y. Hopen, and R. L. Phillips, "Theory of optical scintillation: Gaussian-beam wave model," Waves Random Media 11, 271-291 (2001).

26. F. Strömqvist Vetelino, B. Clare, K. Corbett, C. Young, K. Grant, and L. Andrews, "Characterizing the propagation path in moderate to strong optical turbulence," Appl. Opt. 45, 35343543 (2006).

27. "Downhill simplex method in multidimensions," Chap. 10.4 in Numerical Recipes in C: The Art of Scientific Computing (19881992), http://www.library.cornell.edu/nr/bookcpdf/c10-4.pdf.

28. R. G. Lane, A. Glindemann, and J. C. Dainty, "Simulation of a Kolmogorov phase screen," Waves Random Media 2, 209-224 (1992).

29. J. Recolons and F. Dios, "Accurate calculation of phase screens for the modeling of laser beam propagation through atmospheric turbulence," in Proc. SPIE 5891, 51-62 (2005). 